\title{
Nonlinear dynamics of fluid flow in low-permeability media
}

\author{
YIFENG WANG ${ }^{1}$ \\ ${ }^{1}$ Sandia National Laboratories, Department of Nuclear Waste \\ Disposal Research \& Analysis, P. O. Box 5800, MS0779, \\ Albuquerque, New Mexico 87185-0779, USA; \\ ywang@sandia.gov
}

Understanding fluid flow and mass transport in low-permeability media is of great importance to the development of unconventional hydrocarbon reservoirs and nuclear waste repositories. In this presentation, we discuss some emergent behaviours of gas and water migration in shale (including compacted clay materials) and salt formations. We show that due to complex hydrological, mechanical and chemical couplings, a fluid flow in such media can exhibit rich nonlinear dynamic behaviours including episodic and chaotic fluid migration and release. Such behaviors can be best demonstrated by the experimental data obtained for gas migration in a water-saturated compacted clay material. A time series analysis has been performed on the data. It is found the embedded dimension for the gas migration phenomenon ranges from 3 to 4 . It is also shown that in the embedded space the flow rate seems to possess an internal structure (a chaotic attractor), i.e., not completely random (white noise), indicating a deterministic chaotic behavior. This is further confirmed by the calculation of the spectrum of Lyapunov exponents for gas inflow. To explain these dynamic behaviours, a chaotic model based on the concept of delay logistic model has been developed. In this model, we show that, given a low permeability of the material, the dominant mechanism for gas migration is first to nucleate a bubble and then push the bubble through the clay matrix through dilation and fracturing. In the wake of bubble movement, matrix compression and fracture healing may also take place. Similarly, field measurements of brine inflow in testing boreholes in salt formations also display episodic brine release. We show that this nonlinear behaviour can arise from the couplings among fluid migration, stress-dependent grain boundary wetting and shear-induced dilatancy of salt.

Sandia National Laboratories is a multi-mission laboratory managed and operated by National Technology and Engineering Solutions of Sandia, LLC., a wholly owned subsidiary of Honeywell International, Inc., for the U.S. Department of Energy's National Nuclear Security Administration under contract DE-NA-0003525. The work was supported by DOE Spent Fuel Waste Science \& Technology (SFWST) Program. 